\title{
Optical Mapping Can Reveal Two-Dimensional Morphology of Atrioventricular Insulation in the Foetal Avian Heart
}

\author{
Veronika Olejnickova ${ }^{1,2}$, Oto Janousek ${ }^{3}$,Marina Ronzhina ${ }^{3}$, Jakub Hejc $^{3}$, Eva Zabrodska ${ }^{1}$, Alena \\ Kvasilova $^{1}$, David Sedmera ${ }^{1,2}$ \\ ${ }^{1}$ First Faculty of Medicine, Charles University, Prague, Czech Republic \\ ${ }^{2}$ Instiute of Physiology, Czech Academy of Sciences, Prague, Czech Republic \\ ${ }^{3}$ Department of Biomedical Engineering, Brno University of Technology, Brno, Czech Republic
}

\begin{abstract}
Syndromes of ventricular pre-excitation or atrioventricular $(A V)$ re-entry are believed to results from inappropriate formation of the $A V$ junction (AVJ) during prenatal development. However, technical limitations pose challenge to study foetal heart electrophysiology and are hampering understanding formation of accessory pathways (APs) bypassing AVJ. Here, we present a novel approach of retrograde perfusion for optical mapping of chicken foetal heart. Together with embryonic heart mapping, we were able to investigate AVJ electrical remodelling during the entire prenatal period. AV electrical insulation was observed concomitant to functional deployment of the conduction system at the time of ventricular septation. However, persistence of ectopic AV electrical continuity was still detected in postseptation foetal hearts. APs registered in $40 \%$ of prenatal day 13 (localised in the central anterior or left posterior side) were progressively resolved and none were detected on prenatal day 18. The optical mapping approach thus proved its ability to track AVJ electrical remodelling during whole prenatal chicken development and spatiotemporally localise electrically active APs.
\end{abstract}

\section{Introduction}

Syndrome of pre-excitation represents clinical consequence of inappropriate AV insulation process. In this case, the APs that bypass AV grove allow premature ventricular excitation and could proceed to AV re-entrant tachycardia (AVRT)[1]. Beside the fact that AVRT is a common form of supraventricular tachycardia in children and adult [2, 8], the mechanisms of APs formation is poorly understood. Its prenatal origin as a remnant of initially myocardial AVJ is generally accepted [3]. The AVJ myocardial continuity is normally disrupted by insulating fibrous skeleton, timely coinciding with functional deployment of conduction system along completing ventricular septum. However, impulse propagation through persistent accessory myocardial AV connection has been shown rarely possible even in the late chicken foetus [3]. The inability to visualize activation in post-septated foetal stages limits understanding mechanisms responsible for abnormal APs persistence and electrical behaviour in the late foetal development. Here, we used novel approach of the retrogradely perfused chicken heart for optical mapping conduction through AVJ in the late prenatal stages. Providing two dimensional information about occurrence, exact location and characterisation of APs behaviour could importantly enhance understanding of AVJ abnormal remodelling in the clinical syndromes of preexcitation. Together with maps from embryonic stages, we were able to complete understanding of whole prenatal AVJ electrical development.

\section{Methods}

\section{Isolated heart preparation}

All the animal experiments were performed in accordance with the Czech law governing animal care and experimentation, and were approved by the appropriate institutional committee.

Fertilized White Leghorn chicken eggs were incubated to the desired prenatal day as described previously [4]. The embryos, prenatal day $4(n=14)$ and day $7(n=17)$, and foetuses, prenatal day $13(n=7)$ and $18(n=5)$, were carefully removed from the egg and hearts were rapidly dissected in the ice-cold Tyrode solution $\left(\mathrm{CaCl}_{2} 1.1\right.$ 
mmol/l, glucose $11 \mathrm{mmol} / \mathrm{l}$, HEPES 5 mmol/l; pH7.4). Activation pattern of the embryonic hearts were optically mapped as described previously [4].

\section{Optical mapping of the foetal hearts}

Since nutrition via coronary artery takes place in late prenatal development, the supplementation by diffusion is no longer able to meet increasing metabolic demands. To further track AVJ electrical remodelling, we optimize our previously described retrograde perfusion set-up [5], for optical mapping of the chicken foetal hearts. Briefly, the hearts were cannulated through the aorta and right and left brachiocephalic arteries were separately ligated to avoid perfusate leaking. The hearts were retrogradely perfused in a custom-made perfusion circuit by Tyrode's solution aerated with $100 \%$ oxygen at temperature $37^{\circ} \mathrm{C}$ (Fig. 1). After 5-10 minutes of stabilisation, the hearts were stained by voltage sensitive dye di-4-ANNEPS (Thermo-Fisher Scientific; $500 \mu \mathrm{l}$ of $0,125 \%$ stock diluted in DMSO) slowly injected to the perfusion circuit for 5-7 minutes. Blebbistatin (Sigma-Aldrich) was subsequently injected to the circuit $(25 \mu l$ of $0,125 \%$ stock diluted in DMSO)

Imaging was performed using the ULTIMA L camera (SciMedia) under a 2x, 0.14NA objective lens (effective pixel size $80 \mu \mathrm{m})$ with water immersion lens cap (Olympus) on a fixed-stage BX51 WI epifluorescence microscope (Olympus) equipped with a $150 \mathrm{~W}$ Xe arc lamp (Cairn) and an appropriate wide green filter set. Optical action potentials were recorded at the spontaneous rhythm from the anterior as well as posterior epicardial surface to provide detailed spatial information about APs localization. To characterize pattern of APs electrical utilization, the sampling frequency were 1 or 2 $\mathrm{kHz}$.
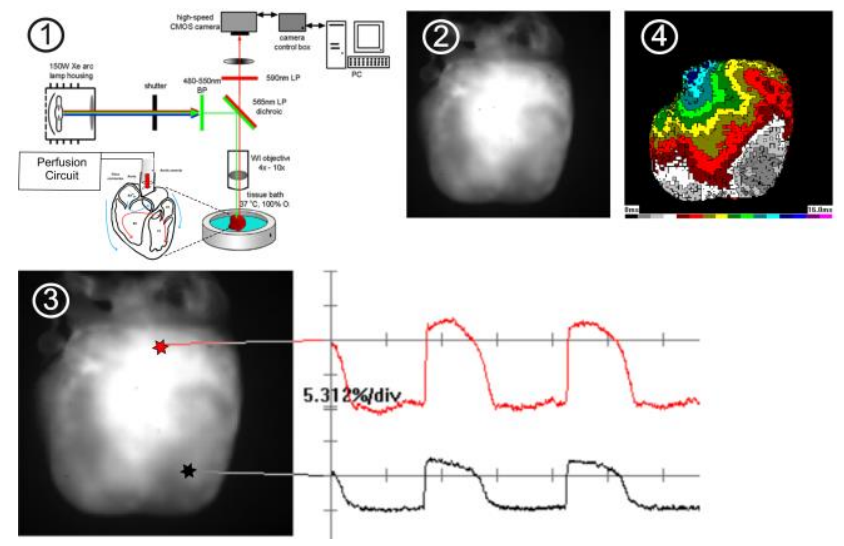

Figure 1.Illustration of the set-up for optical mapping of the chicken foetal heart.

\section{Data processing}

The data was band-pass filtered and processed using a $3 \times 3$ median filter to reduce noise. The first derivative was then numerically calculated, and its peak was used to detect pixel activation time. Spatio-temporal activation maps (Fig. 1) were then constructed as the isochronal maps in the BV_Ana software (SciMedia).

\section{Optical maps analysis}

Ventricular activation patterns were interpreted in conjunction with morphology as discussed previously, and categorized accordingly [4]. Occurrence of APs, their particular location and temporal pattern of utilization were subsequently analyzed. The differences in the frequency of the ventricular activation patterns at each prenatal day were analyzed using Person's Chi-square test. The differences in ventricular activation times were compared using unpaired two-tailed Student's t-test. Pvalues below 0.05 were considered significant.

\section{Results}

Impulse propagation through the AVJ and ventricular activation patterns were successfully optically mapped during the whole prenatal chicken development. In the embryonic hearts, the changes in the immature base-toapex pattern showed transition to mature apex-first in the time of ventricular septation at the prenatal day 7 (Fig. 2 and Fig. 3). This transition of ventricular activation sequence corresponded with ventricular conduction system deployment and shortening of total ventricular activation time (Fig. 3)

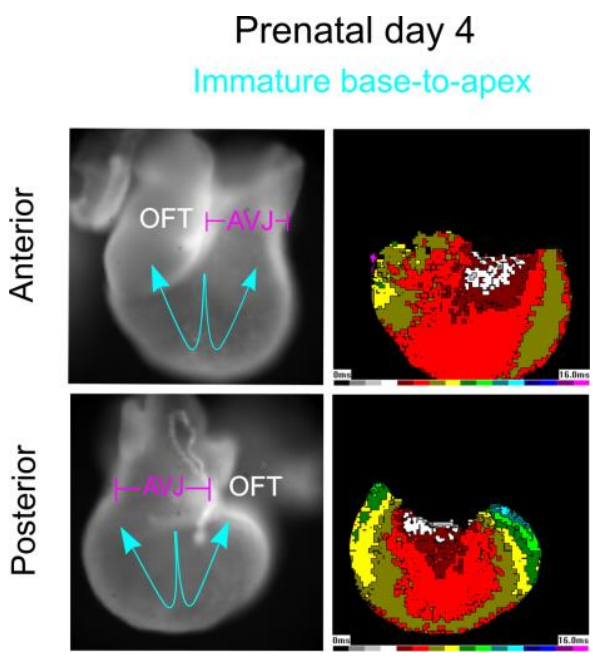

Figure 2. Immature base-to-apex ventricular activation pattern in the prenatal day 4 is going along developing ventricular septum in the anterior and posterior side; AVJ, atrio-ventricular junction; OFT, outflow tract. 
From all retrogradely perfused foetal hearts we were able to obtain activation map (Fig. 4). This was impossible from any hearts loaded by di-4-ANNEPS by diffusion alone (data are not showed). Persistence of AV electrical continuity was still detected in post-septated heart. APs were observed in the $30 \%$ of hearts from prenatal day 13

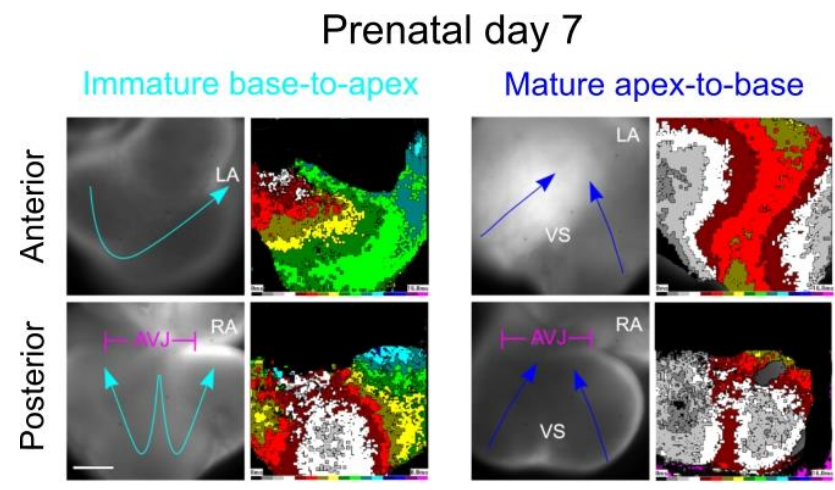

$(n=7)$. The APs were localised in the central anterior or left posterior epicardial surface (Fig. 4). No detected APs in prenatal day $18(\mathrm{n}=5)$ indicate progressive functional remodelling or eventual resolving of these additional AV connections in the later foetal heart development (Fig. 4).

Figure 3. Transition of immature base-to-apex ventricular activation pattern to mature apex-first sequence observed in the prenatal day 7 (left panel) was connected with the shortening of total epicardial activation time recorded at the same prenatal day (right panel); AVJ, atrio-ventricular junction; ED, embryonic day, OFT, outflow tract; VS, ventricular septum; LA, left atrium; RA, right atrium; scale bar $500 \mu \mathrm{m}$.

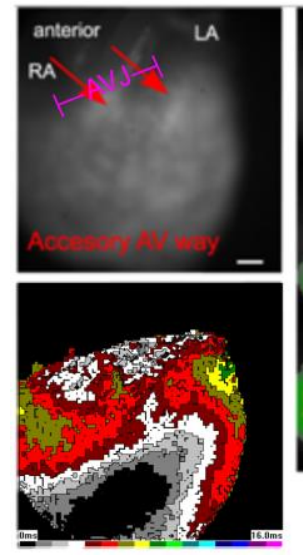

Figure 4. The example of the electrically active AP observed in the prenatal day 13 (upper panels). In this case, the AP was localised in the central anterior surface around the aortic and pulmonary root (red arrow in the left summary map). The pattern of the conduction through AP is shown by white arrows in the time scaled optical map. Example of the fully insulated AVJ in the prenatal day 18 is shown in the right panel; LA, left atrium; RA, right atrium; scale bar $500 \mu \mathrm{m}$.

\section{Discussion}

In this study we used novel approach of retrogradely perfused chicken heart to optically map electrical remodelling of the AV connection in the foetal stages. Beside the fact that persistent $\mathrm{AV}$ connection were

\section{Ventricular activation time}

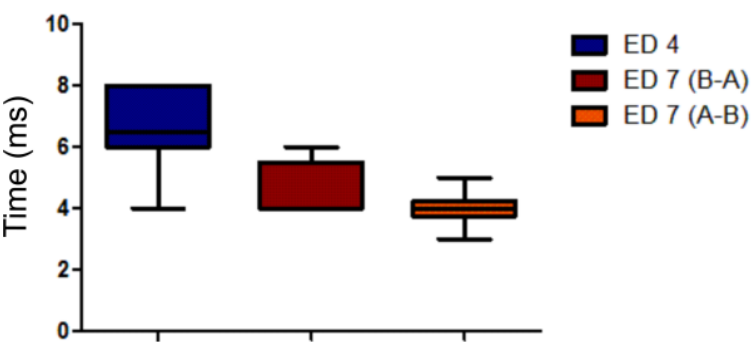


development of mature AVJ $[1,3,6]$ as well as formation of undesirable additional AV bypasses [7]. Although, the role of the apoptosis and fibrotic invagination in AVJ electrical maturation were recently described in our laboratory [8], the understanding of this complex process is far from complete. The inability to connect AVJ morphology with electrophysiological behavior represents main limitation in study underlining mechanisms APs resolving. Presented method allowed us to visualise and spatiotemporally characterise persistence of APs in the foetal development. Moreover, it provides two dimensional information about APs distribution that could enhance understanding of APs persistence in location typical for clinical syndromes of pre-excitation.

Taken together with maps from embryonic stages, we were able to investigate the whole prenatal process of AVJ electrical remodelling.

\section{Conclusion and Perspectives}

This work illustrates the unique capabilities of optical mapping to track AVJ electrical remodelling throughout whole chicken heart development. The APs functional characterisation in the foetal stages is critical for understanding of additional AV connections persistence in the clinical syndromes of pre-excitation and mechanisms of its spontaneous developmental resolution.

Even though the retrogradely perfused foetal chicken heart showed invaluable abilities for APs investigation, the authors believe that this approach could be further enhanced in a few ways. First, since the APs might not necessarily be utilized under normal conditions, their persistence could be unmasked by application of drugs prolonging AV conduction such as adenosine [3]. This manipulation could be particularly useful in the study of mechanisms of APs functional resolving during late foetal stages. Second, for the study of mentioned mechanisms would be optimal to investigate the same heart repeatedly. This approach could be possible with utilisation of invitro cultured chicken heart already established in our laboratory [4]. Third, since the frequency of the APs persistence in the presented work was not high enough and since the APs are often clinically connected with structural AVJ deformation, we plan additionally utilize model of epicardial inhibition [8] and model of ventricular septal defect. Both models, already established in our laboratory, could enhance underling mechanisms that can lead to APs formation in more detail. Last, with our experimental set-up, we investigated electrical behaviour of APs bypassing the AV grove from the epicardial surface only. Using different VSD with deeper penetration ability or precise dissection the AVJ region could be further possible to study APs connecting atria with ventricles in the deeper structures of AVJ.

\section{Acknowledgments}

Supported by the grants CZ.02.1.01/0.0/0.0/16_013/0 001775:Czech-BioImaging funded by OP RDE.

\section{References}

[1] Akazawa, H., and Komuro, I. "Navigational error in the heart leads to premature ventricular excitation." J Clin. Invest, vol. 121, no. 2, pp. 513-516, Feb. 2011.

[2] Hahurij, N.D., Gittenberger-De Groot, A., Kolditz D.P., Bökenkamp, R., Schalij, M.J., Poelmann, R.E., and Blom, N.A. "Accessory atrioventricular myocardial connections in the developing human heart : relevance for perinatal supraventricular tachycardias". Circulation, Vol. 117, no. 22, pp. 2850-2858, 2008.

[3] Kolditz, D.P., Wijffels, M.C., Blom, N.A., Markwald, R.R., Schalij, M.J., and Gittenberger-de Groot, A.C. "Persistence of functional atrioventricular accessory pathways in postseptated embryonic avian hearts: implications for morphogenesis and functional maturation of the cardiac conduction system". Circulation, vol. 115, no. 1, pp. 17-26, Jan. 2007

[4] Sankova, B., Machalek, J., and Sedmera D. "Effects of mechanical loading on early conduction system differentiation in the chick". Am J Physiol. Heart. Circ. Physiol. Vol. 298, no. 5, H1571-1576, May. 2010

[5] Olejnickova, V., Novakova, M., and Provaznik, I. " Isolated heart models: cardiovascular system studies and technological advances" Med. Biol. Eng. Comput. Vol. 53, no.7, pp. 669-678, Jul. 2015.

[6] Rentschler, S., Vaidya, D.M., Tamaddon, H., Degenhardt, K., Sassoon, D., Morley, G.E., Jalife, J., Fishman, G.I. "Visualization and functional characterization of the developing murine cardiac conduction system", Development. Vol. 128, no. 10, pp. 1785-1792, May. 2001.

[7] Jongbloed, M.R., Wijffels, M.C., Schalij, M.J., Blom, N.A., Poelmann, R.E., van der Laarse, A., Mentink, M.M, Fishman, G.I., and Gittenberger-de Groot, A.C. "Development of the right ventricular inflow tract and moderator band: a possible morphological and functional explanation for Mahaim tachycardia." Circ Res. Vol. 96, no. 7, pp. 776-783. Apr. 2005

[8] Vicente-Steijn, R., Sedmera, D., Blom, N.A., Jongbloed, M., Kvasilova, A., and Nanka, O., "Apoptosis and epicardial contributions act as complementary factors in remodeling of the atrioventricular canal myocardium and atrioventricular conduction patterns in the embryonic chick heart." Dev. Dyn., Vol 247, no. 9, pp. 1033-1042, Sep. 2018

Address for correspondence:

Veronika Olejnickova

First Faculty of Medicine, Charles University, U Nemocnice 3, Prague, Czech Republic, 12800

veronika.olejnickova@1f1.cuni.cz 